\title{
NSSTC capabilities and the GNSSaS satellite project
}

\begin{abstract}
This communication introduces the National Space Science and Technology Center (NSSTC), established at the United Arab Emirates University (UAEU) in 2016, its main activities and its first satellite project. NSSTC primary research and development areas are: Remote sensing, Space and planetary science, real time embedded systems, in-situ resource utilization, In-orbit propulsion as well as Space communication and satellite based navigation. The latter will be the focus of this communication and in particular the Radio Frequency (RF) signaling technology satellite, GNSSaS. This is a $6 \mathrm{U}$ Cubesat that will be launched in 2021 into a $500-600 \mathrm{~km}$ sun synchronous orbit. It will include an Global Navigation Satellite System (GNSS) augmentation RF signaling demonstrator payload, GNSS reflectometry payload for remote sensing, and a UHF/VHF experiment for measuring the effects of the ionosphere on these RF frequencies. The paper will describe the objectives of this cubesat in more detail and how it ties up with the rest of NSSTC activities.
\end{abstract}

Keywords: The National Space Science and Technology Centre, Global Navigation Satellite System, Global Navigation Satellite augmentation System, radio frequency
Volume 4 Issue 4 - 2020

\author{
Alhashmi K,Al-Emam O, Hasbi AM, Mustapha \\ MI \\ The National Space Science and Technology Center, United Arab \\ Emirates University, UAE
}

Correspondence: Hasbi AM, Research Associate, The National Space Science and Technology Center, $\mathrm{H} 4$ building, United Arab Emirates University, P.O. Box 1555 I,Abu Dhabi, United Arab Emirates,Tel+97/ 7134492, Email alina.hsbi@uaeu.ac.ae

Received: October 17, 2020 | Published: November II, 2020

\section{Abbreviations: ICT-TRA, ICT-Telecommunications regulation authority; UAE, United Arab Emirates; UAEU, United Arab Emirates University; AIT, assembly, integration and test; GNSS, global navigation satellite system; GNSSaS, global navigation satellite augmentation system; RF, radio frequency; UHF, ultra high frequency; MEO, middle earth orbit \\ Introduction to the National Space Science and Technology Centre}

The National Space Science and Technology Centre was established in 2016 with funding support from the ICT-TRA (ICTTelecommunications Regulation Authority) and United Arab Emirates University (UAEU), as well as project based funding from the UAE Space Agency. The centre has a world class assembly, integration and test (AIT) facility as well as first class ground station and control centre with an array of antenna assets covering Ultra High Frequency (UHF), $\mathrm{S}$ and $\mathrm{X}$ Satellite communication bands. The centre is based on the UAEU campus, and is integrated with the university administrative and academic system, which ensures that the centre has close links with all the University colleges that can contribute to its activities relating to developing Space science and technologies. These colleges include the college of Engineering, Information and Computer Technology, Science as well as Law. This strategic positioning of the centre within the University environment, enables it to have access to expert academics and researchers that can contribute and support the centres activities, at the same time allowing the centre to provide a platform for students at all levels and researchers to be engaged in leading edge space science and technology research and development. The centre employs its own expert Space engineering and scientific team that oversee the various projects at the centre that are carried out by young Emirati engineers who are also employed by the centre. The centre also offers opportunities for internships and post docs, as well as MSc and $\mathrm{PhD}$ students to be involved in its projects that span six pillars of research that include Remote sensing, Space and planetary science, real time embedded systems, in-situ resource utilisation, In-orbit propulsion as well as Space communication and satellite based navigation. Each pillar of research is contained within its own dedicated research group and has its own lab facilities. The glue between the research groups are the funded space mission projects whose aim is to develop satellites that can utilise the group's research and development outcomes as well as provide the necessary data for these groups to carry out their research. One such Space mission project is the GNSSaS Radio Frequency (RF) signalling technology demonstrator satellite, which is a satellite development associated with the communication and satellite navigation group. Its aim is to develop Global Navigation Satellite augmentation system (GNSSaS) for the UAE that can enhance the position determination using existing GNSS signalling through innovative augmentation techniques.

\section{The GNSSaS program}

\section{Background and motivation}

The GNSSaS RF signalling technology demonstrator satellite is part of long-term program that will provide a unique opportunity for the UAE to become an actor of a strategic international technology with associated educational, technical development and potential commercial benefits by:

- Demonstrating a new and innovative GNSS augmentation technology by developing the navigation system payload.

- Providing a GNSS augmentation proof of concept for a high positioning accuracy of $<1 \mathrm{~m}$ system with improved availability and resistance to jamming and spoofing.

- Demonstrating that the proposed GNSS augmentation capability is compatible with existing systems.

- The GNSSaS will utilize all of the existing as well as future GNSS constellations to provide an innovative augmentation concept.

- Develop, build, test and launch, and operate the GNSSaS satellite.

- Establish basis, starting with request for frequency filling approvals from regional (UAE TRA) and international regulatory authorities (International Telecommunication Union "ITU") to allow for the broadcasting of GNSS augmentation signals. 
The GNSSaS will support the following UAE Space Agency strategic objectives:

- Develop and guide a world-class Space sector that contributes to a diversified national economy and which supports sustainable development.

- Support Space science research and exploration in support of the advancement of UAE SA Space science, technology and innovation (ST\&I) road map.

- Grow generations of highly skilled UAE nationals to become leaders in Space disciplines by exercise and training over multidisciplinary projects in the field of GNSS.

- Encourage and support the provision of GNSS support services and GNSS based applications that are of high quality and standards as well as providing an opportunity to create new start-ups that can fuel a sustainable technology-based economy in the UAE.

It is hoped that the proposed GNSSaS development will place the UAE in the forefront of stakeholders and acting nations in the domain of navigation and positioning augmentation technologies. In addition, the program will support the development of spacecraft technologies of critical value to the UAE National interests, building up the capabilities and facilities available in the UAE for future programs.

\section{GNSSaS program technical rational}

Figure 1 show the L-band part of the existing GNSS signals, ${ }^{1}$ knowing that also BEIDOU and IRNSS are transmitting GNSS signals in L and S bands. The future GlobalStar GNSS PPP message (Point Precise Positioning) and pilot signals, will be transmitted in S-band. ${ }^{2}$ The next Korean Positioning System will also transmit GNSS signals in $\mathrm{L}$ and $\mathrm{S}$ bands. ${ }^{3}$ It is worth noting that the following governments are also fielding regional augmentations: USA, Europe, China, Russia,
Japan, India, Nigeria and South Korea. ${ }^{4}$ The European Galileo system is introducing a new High Accuracy Service (HAS). ${ }^{5}$ With its L and $\mathrm{S}$ band navigation and timing signals, GNSSaS will therefore be at the forefront of GNSS in term of performances, interoperability and complementarity. These are a fully complementary response to the current pressing market needs, which include:

- Autonomous vehicles which are rapidly emerging and need a viable complementary technology to the on board sensors for localization on the road.

- Drones (UAV) are proliferating and need highly accurate and reliable GPS positioning.

- Precision farming is a global imperative, yet current systems don't always work.

- The need to navigate accurately in urban canyons, where multipath effects caused by GNSS signal reflections reduce prescient where it is most needed.

- The need for robust GNSS cybersecurity to defeat malicious attacks and hacking.

- Growing threat from jamming and spoofing.

Many governmental bodies across the globe have recognized the above needs and have been working towards developing improved anti-jamming, anti-spoofing GNSS countermeasure technologies, with guaranteed availability, high performance, and precision. The existing systems such as GPS, GLONASS, GALILEO and BEIDOU currently provide 5 to $10 \mathrm{~m}$ position accuracy with $95 \%$ confidence, without augmentation. ${ }^{6-8}$ While the modernized GPS frequencies service (e.g. L1C, L2C \& L5) include multi-frequency signals which improve availability, accuracy and robustness. It is also worth noting that Galileo and IRNSS costly and complex atomic clocks used for navigation are sometimes failing in orbit, suggesting demand for simpler, lower-cost alternatives.
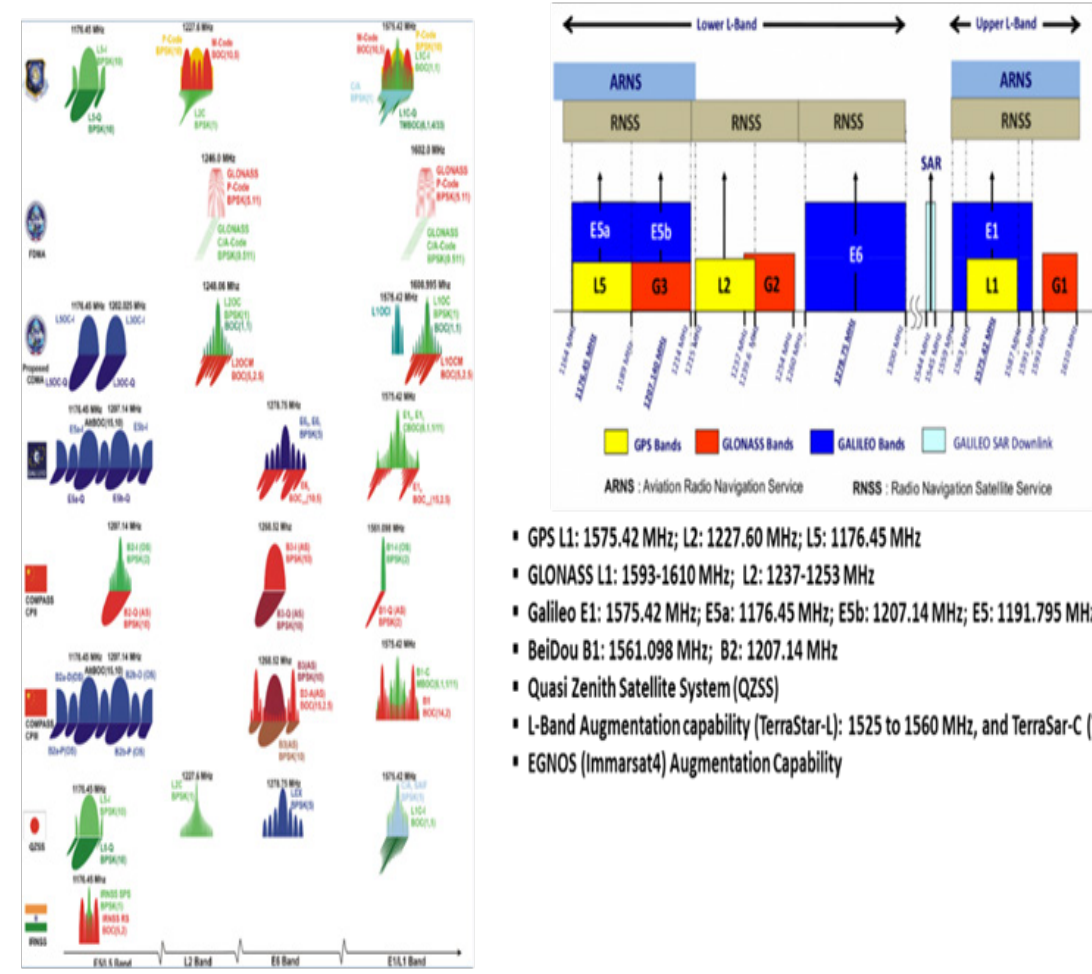

- GPS L1: 1575.42 MHz; L2: 1227.60 MHz; 15: 1176.45 MHz

- GLONASS LI: 1593-1610MHz; 22:1237-1253 MHz

- Galileo E1: 1575.42 MHz; E5: 1176.45 MHz; E5b: 1207.14 MHz; E5: $1191.795 \mathrm{MHz}$;

- BeíDou B1: $1561.098 \mathrm{MHz} ;$ B2: 1207.14 MHz

- Quasi Zenith Satellite System(Q25s)

- L-Band Augmentation capability (Terrastarl): 1525 to $1560 \mathrm{MHz}$, and Terrasar-C (TBO)

- EGNOS (Immarsat4) Augmentation Capability

Figure I Current GNSS Constellations (adapted from Subirana et al., 20I I). 


\section{The GNSSaS RF signalling technology demonstrator satellite}

The GNSSaS RF signalling technology demonstrator satellite is a $6 \mathrm{U}, 2 \times 3 \mathrm{U}$ cubesat, $10 \times 20 \times 30 \mathrm{~cm}$ with a mass of $12 \mathrm{~kg}$, and a power generation capacity of $>25 \mathrm{Wh}$ per orbit. It is implemented using the standard cubesat format where $1 \mathrm{U}$ is $10 \times 10 \times 10 \mathrm{~cm}$. The satellite under development includes deployable solar panels, and utilized UHF and $\mathrm{S}$ bands communication systems for command, telemetry and data. It will be controlled from the ground station and mission control centre at NSSTC. As part of the payload a bespoke L-band four (4) patches antenna array was developed. The Cubesat is expected to be launched in 2021 into a sun synchronous low earth orbit (LEO), 500-600km. The satellite includes a number of payloads all of which relates in one way or another to GNSS. These include the primary NSSTC developed GNSSaS payload relating to the transmission of the GNSS augmentation signals in both GNSS L and S bands.

The GNSSaS RF signalling technology demonstrator satellite payloads are based on Software Defined Radio (SDR) platforms that are designed to provide the necessary flexibility that will enable the performance of various experiments by uploading new software and firmware. .Re-programmability is an important feature and essential when developing a versatile technology demonstrator satellite that can be adapted to various needs depending on the outcome of its baseline experiments. This will ensure that the satellite's payloads content can be updated throughout the missions planned three (3) year life time to remain current and relevant to changing requirements. NSSTC is developing modular on-board Command and Data Handling System (CDHS) and Attitude Determination and Control System (ADCS) that will be implemented on this satellite with the flexibility to be easily customized and adapted to various NSSTC Space missions, but more importantly to be able to update during the satellite operational life time. The CDHS and AOCS modular software developments are carried out as part of UAE Space Agency funded projects and cofunded by NSSTC.

\section{The GNSSaS mission a platform for collaboration}

The GNSSaS RF signalling technology demonstrator satellite is designed and developed by the NSSTC engineers in collaboration with GomSpace who is providing the spacecraft platform subsystems' hardware units. The project also included involvement of students performing their final year undergraduate projects, as well as those doing their internship. The satellite AIT will also take place at the NSSTC bespoke AIT facilities.

In addition to the primary GNSSaS payload, there are in addition a number of secondary payloads: One provided by Syrlinks to demonstrate their in-orbit GNSS receiver system, another provided by CNES which is related to high precision time and orbital ephemeris real time determination system using signals from MEO GNSS satellites. Last but not least a secondary payload from the Universitat Politècnica de Catalunya UPC-Barcelona Tech (Spain) (https:// nanosatlab.upc.edu/en), which is an L5-band GNSS-Reflectometry remote sensing experiment, and a UHF/VHF bands signal quality monitoring experiment for ionospheric scintillation studies

\section{Conclusion}

The proposed GNSSaS RF Signaling Technology Demonstrator is the first stage of a program to develop GNSS augmentation using Satellites in LEO. The ultimate aim is to assess and evaluate the feasibility of having a constellation of small satellites LEO that would transmit GNSS augmentation Signals. The objective here is to provide an improved performance and resilience at lower cost relative to other existing GNSS combined with GNSS augmentation systems. GNSSaS can potentially offer:

- A GNSS innovative solution that has not yet been implemented.

- Compatibility with existing GNSS (GPS, GLONASS, BEIDOU, etc.

- Improved positioning accuracy from existing GNSS of meters to centimetres accuracy.

- Resilience to jamming and spoofing, including cybersecurity protection measures.

- A nationally owned and operated UAE GNSS augmentation system.

- Build a cornerstone of the UAE government strategy for a "UAE Digital and Knowledge based economy".

In conclusion it is clear that the secondary payloads' contribution from world renowned Space Science and Technology players is a clear recognition of NSSTC's ability and expert capabilities to develop complex space missions, as well as a vindication that NSSTC's research programs is pushing in the right direction exploring pioneering approaches to find solutions to topical challenges in the domain of high precision GNSS geolocation, assessing atmospheric effects on RF communication signal of high importance to today's space based applications, as well innovative approaches to remote sensing using GNSS reflectometry. These hosted payloads offer a collaborative opportunity and a shared benefit for all those involved. The aim is that the data resulting from this satellite mission will be processed and analyzed by NSSTC specialists and their partners as well as the MSc and PhD students that will be engaged with NSSTC to carry out the research as part of their degrees at UAEU.

\section{Acknowledgments}

The NSSTC wishes to express their gratitude to the ICT-TRA fund, UAE University (UAEU) and UAE Space Agency (UAESA) for establishing and funding the center. NSSTC would like to acknowledge the funding and support from the UAESA for the GNSSaS project, which has been instrumental in enabling this research and development to take place. The authors wish to thank CNES, Syrlinks and UPC for providing the hosted payloads that will significantly increase the scientific return from the GNSSaS mission. Special appreciation goes to Eng Jean-Luc Issler from CNES and Dr Adriano from UPC for their valuable technical advice and continued support throughout the development of the GNSSaS project. It goes without say that the GNSSaS project would not have been possible to realise without the hard work and dedication of all the project team members at NSSTC.

\section{Conflicts of interest}

Authors declare that there is no conflict of interest.

\section{References}

1. Subirana JS, Zornoza JMJ, Hernandez-Pajares M. GNSS signal. ESA Navipedia; 2011.

2. Mateu I, Boulanger C, Issler J, et al. Exploration of possible GNSS signals in S-band. GNSS 2009 22nd International Technical Meeting of The Satellite Division of the Institute of Navigation (ION GNSS 2009). Savannah: United States; 2009. 1573-1587 p. 
3. GPS World Staff. Korea will launch its own satellite positioning system GPS World; 2018.

4. Lawal LS, Chatwin CR. A Review of GNSS and Augmentation Systems. Journal of Electrical and Electronics Engineering. 2019;5(3):1-21.

5. Borio D, Senni T, Hernandez IF. Galileo's High Accuracy Service: Field Experimentation of Data Dissemination Schemes. Inside GNSS; 2020.

6. Odolinski R, Teunissen PJG, Odijk D. First combined COMPASS/ BeiDou-2 and GPS positioningresults in Australia. Part I: singlereceiver and relative code-onlypositioning. Journal of Spatial Science. 2014;59(1):3-24.
7. Angrisano A, Gaglione S, Gioia C, e al. Benefit of the NeQuick Galileo version in GNSS single-point positioning. International Journal of Navigation and Observation. 2013;2013:302947.

8. Nik SA, Petovello MG. Implementation of a Dual-Frequency GLONASS and GPS L1 C/A Software Receiver. The Journal of Navigation. 2010;63(2):269-287. 